\title{
RNA viral vectors for improved Agrobacterium-mediated transient expression of heterologous proteins in Nicotiana benthamiana cell suspensions and hairy roots
}

\author{
Jeffrey S Larsen and Wayne R Curtis
}

\begin{abstract}
Background: Plant cell suspensions and hairy root cultures represent scalable protein expression platforms. Low protein product titers have thus far limited the application of transient protein expression in these hosts. The objective of this work was to overcome this limitation by harnessing A. tumefaciens to deliver replicating and non-replicating RNA viral vectors in plant tissue co-cultures.

Results: Replicating vectors derived from Potato virus $X(P V X)$ and Tobacco rattle virus (TRV) were modified to contain the reporter gene $\beta$-glucuronidase (GUS) with a plant intron to prevent bacterial expression. In cell suspensions, a minimal PVX vector retaining only the viral RNA polymerase gene yielded 6.6-fold more GUS than an analogous full-length PVX vector. Transient co-expression of the minimal PVX vector with P19 of Tomato bushy stunt virus or HC-Pro of Tobacco etch virus to suppress post-transcriptional gene silencing increased GUS expression by 44 and $83 \%$, respectively. A non-replicating vector containing a leader sequence from Cowpea mosaic virus (CPMV-HT) modified for enhanced translation led to 70\% higher transient GUS expression than a control treatment. In hairy roots, a TRV vector capable of systemic movement increased GUS accumulation by 150-fold relative to the analogous PVX vector. Histochemical staining for GUS in TRV-infected hairy roots revealed the capacity for achieving even higher productivity per unit biomass.

Conclusions: For the first time, replicating PVX vectors and a non-replicating CPMV-HT vector were successfully applied toward transient heterologous protein expression in cell suspensions. A replicating TRV vector achieved transient GUS expression levels in hairy roots more than an order of magnitude higher than the highest level previously reported with a viral vector delivered by A. tumefaciens.
\end{abstract}

Keywords: Plant tissue culture, Gene silencing, Viral vectors, Hairy roots, Plant cell suspensions, Potato virus X, Tobacco rattle virus, Transient protein expression, Agrobacterium tumefaciens

\section{Background}

While a plethora of viral vectors have recently been developed for agroinfiltration of plants [1-4], there are few reports of successful application of these vectors to in vitro systems [5]. Plant cell suspensions and hairy roots combine the advantages inherent to plants with the environmental control, scalability, short production cycles, and containment of tissues cultured in bioreactors.

\footnotetext{
* Correspondence: wrc2@psu.edu

Department of Chemical Engineering, The Pennsylvania State University, University Park, Pennsylvania, 16802, USA
}

The controlled and aseptic nature of these in vitro systems makes them readily compliant with good manufacturing practice and suitable for the production of therapeutic proteins [6].

Plant cell suspensions are composed of individual and small aggregates of plant cells cultured in a liquid medium containing hormones that promote proliferation and de-differentiation. Cell suspensions are readily scalable volumetrically in bioreactors by principles already developed for microbial and mammalian cell cultures [7]. In fact, plant cell suspensions have been scaled up to $75,000 \mathrm{~L}$ for the production of valuable 
secondary metabolites [8]. They are also amenable to pilot-scale culture in low capital cost bioreactors [9].

Unlike plant cell suspensions, hairy root cultures consist of a tissue with multiple cell types. Hairy roots develop as the consequence of the transfer of genetic information from Agrobacterium rhizogenes, a gram-negative soil bacterium, to a host plant. This 'natural' process leads to the emergence of genetically transformed hairy roots at the site of infection on the plant. The hairy root phenotype is characterized by fast, hormone-independent growth, lack of geotropism, lateral branching and genetic stability [10]. Hairy root cultures are also scalable in bioreactors [11].

Both plants and plant tissue cultures are amenable to a rapid, scalable and low-cost gene delivery method using recombinant clones of Agrobacterium tumefaciens. When co-cultured with plant tissues $A$. tumefaciens can efficiently mediate the transfer of heterologous T-DNA to the nucleus of the plant cells. Transient expression of this episomal DNA results in a temporal peak in heterologous protein expression in a matter of days. Consequently, transient expression is suitable for manufacturing proteins that require very short lead times. For example, the first doses of a plant-made influenza vaccine can be produced within three weeks of the release of sequence information for new pandemic strains [12]. In contrast, months are required to generate and select productive transgenic cell lines which can accumulate mutations in the transgenes over time when maintained by serial subculture [13].

In the case of RNA viral vectors, $A$. tumefaciens delivers cDNA that is transcribed to the viral RNA genome in the nucleus and exported to the cytoplasm. Subsequent RNA replication cycles amplify both viral and heterologous genes. Concurrent with genome replication, viral polymerases also synthesize subgenomic RNAs that direct target protein translation. These double-stranded RNA intermediates can trigger posttranscriptional gene silencing (PTGS). Subsequently, homologous RNA transcripts may fail to accumulate due to sequence-specific targeting and destruction [14]. The 19-kDa gene product (P19) from Tomato bushy stunt virus is a potent suppressor of PTGS triggered by transient expression in plants [15].

PVX has a monopartite plus-sense RNA genome encoding five open reading frames (ORFs). The first ORF encodes the RNA-dependent RNA polymerase required for viral replication. The central three overlapping ORFs, known as the triple gene block (TGB), are required for local movement of the virus to neighboring cells. The final ORF encodes the PVX coat protein (CP) which is required for virion assembly, cell-to-cell infection via plasmodesmata, and systemic movement through the vasculature. The viral polymerase is translated directly from the viral RNA transcript and synthesizes the subgenomic plus-sense RNAs from which the other four
ORFs are translated [16,17]. In plants PVX has been utilized as a full-length expression vector capable of infecting distal tissues [18-20] as well as a deleted vector lacking viral genes essential for local and systemic movement [21].

The TRV genome is divided into two plus-sense singlestranded RNAs that are separately encapsidated. RNA-1 encodes the viral proteins responsible for replication and movement, while RNA-2 encodes the viral coat protein and the transgene in place of two non-structural ORFs required for nematode transmission [22]. The smaller RNA-2 is nonessential for systemic infection of plants, such that it can be extensively modified without negatively affecting virus stability [23]. Tobraviruses such as TRV have adapted for efficient replication and movement in the roots of infected plants. Here they invade meristematic tissues, such as growing tips, to be ingested by specific nematode transmission vectors [24]. While replicating TRV and PVX vectors were reported to express GFP to similar levels in systemically infected leaves of $N$. benthamiana, infection with TRV resulted in 10 to 25 -fold more GFP accumulation in the roots of these plants. This disparity in GFP expression was attributed to the root tropism of TRV [23].

While PVX and TRV vectors require viral replication to achieve high-level expression, the CPMV-HT expression system relies on enhanced translation in the absence of viral replication [1]. The pEAQ-HT vector based on this system includes the P19 gene on the same T-DNA as the target gene [25]. In a side-by-side comparison, the non-replicating 'hyper-translatable' CPMV-HT expression system yielded five times more anti-HIV monoclonal antibody than the analogous replicating deleted version of CPMV RNA-2, without detrimentally impacting post-translational modifications [26].

In this study we evaluated the three RNA viral vectors described above for their potential to achieve rapid, high-level Agrobacterium-mediated transient protein expression in $N$. benthamiana cell suspensions and hairy roots.

\section{Methods}

\section{Plants and plant tissue cultures}

Callus cultures of wild-type $N$. benthamiana were generated by placing leaf and petiole tissue explants from aseptically-grown seedlings onto MSG media solidified with $6 \mathrm{~g} / \mathrm{L}$ agar. MSG is a derivative of MS salts media [27], containing $25 \mathrm{~g} / \mathrm{L}$ sucrose, 1X B5 vitamins [28], $0.5 \mathrm{mg} / \mathrm{L}$ 2,4-dichlorophenoxyacetic acid (2,4-D), and $0.2 \mathrm{mg} / \mathrm{L}$ kinetin with the $\mathrm{pH}$ adjusted to 5.7. Following callus induction, liquid cell suspension cultures were subsequently maintained on MS media with $0.2 \mathrm{mg} / \mathrm{L}$ 2,4-D and $25 \mathrm{~g} / \mathrm{L}$ sucrose $\mathrm{pH}$ adjusted to 5.5 in $500-\mathrm{mL}$ Erlenmeyer flasks. Suspensions were serial subcultured 
every 14 days by transferring $20 \mathrm{~mL}$ into $100 \mathrm{~mL}$ of fresh media and returning $20 \mathrm{~mL}$ to the original flask to achieve a final volume of $100 \mathrm{~mL}$. Callus generated from four individual plants was used to establish four cell lines consisting of fine liquid suspensions. Suspensions were screened by co-culture with $A$. tumefaciens harboring pBY031-I1. A single cell line was selected and used for subsequent experiments based on good growth and superior transient GUS expression after 4 days of co-culture as determined by the qualitative assay.

Hairy root cultures were generated by transforming aseptically grown $N$. benthamiana seedlings with $A$. rhizogenes [ATCC:15834]. Individual roots that grew from different wound sites exhibiting the hairy root phenotype of fast, highly-branched, and hormone-independent growth were cultured separately and cured of bacteria on B5 medium supplemented with $300 \mathrm{mg} / \mathrm{L}$ cefotaxime. A single root clone was selected based on good growth and used for subsequent experimental work. Hairy root cultures were maintained on B5 medium with $25 \mathrm{~g} / \mathrm{L}$ sucrose $\mathrm{pH}$ adjusted to 5.5 in 125-mL Erlenmeyer flasks and were serial subcultured every 14 days by transferring $\sim 500 \mathrm{mg}$ into $50 \mathrm{~mL}$ of fresh media [28]. All cell suspensions and hairy root cultures were incubated at $25^{\circ} \mathrm{C}$ in the dark on a 120 RPM orbital shaker with a $2.5 \mathrm{~cm}$ stroke.

Nicotiana benthamiana plants were grown from seed under fluorescent lighting with a 16 hour photoperiod in an incubator maintained at $23^{\circ} \mathrm{C}$. Plants were grown in $10 \mathrm{~cm}$ square pots with Miracle-Gro potting mix containing sphagnum peat moss and perlite in a 3:1 ratio supplemented with dolomitic lime at the recommended concentration of $5.3 \mathrm{~g} / \mathrm{dm}^{3}$. Plants were fertilized with liquid Dyna-Gro 7-9-5 at each watering at the rate recommended for outdoor plants $(0.0326 \%$ by volume).

\section{Co-culture of plant tissues with Agrobacterium tumefaciens} Plant cell suspensions were prepared for an experiment by adding $5 \mathrm{~mL}$ of 2-week-old suspension culture into $15 \mathrm{~mL}$ of MS media supplemented with $0.2 \mathrm{mg} / \mathrm{L} \mathrm{2,4-D,}$ $25 \mathrm{~g} / \mathrm{L}$ sucrose and $2.5 \mathrm{mM} \mathrm{KH_{2 }} \mathrm{PO}_{4}$ in a 125 -mL flask with a loose foil closure and no sponge plug. Within two hours, A. tumefaciens clones harboring the appropriate plasmids were then added to initiate co-culture. Root cultures were initiated by adding $\sim 500 \mathrm{mg}$ fresh weight of 2 -week-old roots into $25 \mathrm{~mL}$ of B5 media in a $125-\mathrm{mL}$ flask with a loose foil closure. Unless noted otherwise, root cultures were incubated under standard conditions for 5 days prior to initiation of co-culture.

The A. tumefaciens strain used for co-culture with plant suspensions was the cysteine auxotroph C58:: pEHA105/Cys32, which was generated by transposon mutagenesis [29]. Growth of the bacteria was monitored in liquid culture on the basis of measurements of absorbance at $600 \mathrm{~nm}\left(\mathrm{OD}_{600}\right)$. A. tumefaciens clones harboring the appropriate plasmids were cultured individually in Luria-Bertani (LB) media supplemented with $50 \mathrm{mg} / \mathrm{L}$ kanamycin in $25 \times 150 \mathrm{~mm}$ culture tubes on a $1.9 \mathrm{~cm}$ stroke rotary shaker at $250 \mathrm{RPM}$ and at $25^{\circ} \mathrm{C}$. Cryogenic stocks of each Agrobacterium clone were used to inoculate $2 \mathrm{~mL} \mathrm{LB}$ media with selection. These cultures were then grown overnight and used to quantitatively inoculate $5 \mathrm{~mL}$ aliquots of fresh $\mathrm{LB}$ media with selection to achieve the desired bacterial density based on an additional 6 to 8 hours of growth. Once the A. tumefaciens cultures reached an exponential growth phase $\left(\mathrm{OD}_{600}\right.$ 1) they were pelleted by centrifugation at 3000 RCF for $7 \mathrm{~min}$, washed, and resuspended in root or cell suspension medium to an $\mathrm{OD}_{600}$ of approximately 1.0. Each bacterial culture was added to the plant tissue culture flasks at the equivalent of $1 \%$ of the working culture volume at an $\mathrm{OD}_{600}$ of 1.0. In all experiments involving pTRV2-GUS, a separate $A$. tumefaciens clone harboring PTRV1 was always co-inoculated in a 1:1 ratio.

\section{Histochemical GUS detection and quantification}

After six days of co-culture, GUS expression in each flask was evaluated qualitatively by histochemical staining with X-Gluc substrate [30]. Approximately $50 \mathrm{mg}$ fresh weight of plant cell suspension filter cake was added to $75 \mu \mathrm{L}$ of isotonic solution $(6 \mathrm{~g} / \mathrm{L}$ sodium chloride) in a flat-bottom 96-well microtiter plate. $100 \mathrm{mg}$ fresh weight of roots (blotted dry) was collected from the outside edge of the root mat and transferred to a $1.5 \mathrm{~mL}$ microcentrifuge tube. Freshly harvested samples were incubated at room temperature with $150 \mu \mathrm{L}$ (cells) or $200 \mu \mathrm{L}$ (roots) of X-Gluc staining buffer (2 mM 5bromo-4-chloro-3-indolyl $\beta$-D-glucuronic acid, $10 \mathrm{mM}$ EDTA, $0.1 \%$ Triton $\mathrm{X}-100,100 \mathrm{mM}$ sodium phosphate buffer at $\mathrm{pH} 7.0,0.5 \mathrm{mM}$ potassium ferricyanide, $0.5 \mathrm{mM}$ potassium ferrocyanide). $200 \mathrm{mg}$ samples were also collected for the quantitative MUG assay and stored at $-20^{\circ} \mathrm{C}$ prior to processing.

The yield of GUS was quantified through a fluorometric assay. Protein was extracted by the addition of extraction buffer $(333 \mathrm{mg} / \mathrm{mL}$ silicon carbide, $50 \mathrm{mM}$ sodium phosphate buffer at $\mathrm{pH} 7.0,10 \mathrm{mM}$ sodium EDTA, $0.1 \%$ Triton $\mathrm{X}-100,0.1 \%$ sodium lauryl sulfate, $10 \mathrm{mM}$ 2-mercaptoethanol) at $0.45 \mathrm{~mL} / \mathrm{g}$ fresh weight roots or cells, or $2 \mathrm{~mL} / \mathrm{g}$ fresh weight leaf tissue. Tissue was frozen in liquid nitrogen and homogenized with a sterile polypropylene mini-pestle (Sigma-Aldrich, part number Z359947) in a $1.5 \mathrm{~mL}$ microcentrifuge tube at 60 RPM for 80 seconds. Homogenized samples were centrifuged at $16,000 \mathrm{RCF}$ for 10 minutes at $4^{\circ} \mathrm{C}$. The total soluble protein (TSP) in the supernatant was quantified using a modified Bradford assay with bovine serum albumin as the protein standard [31]. Samples were then diluted with extraction buffer (silicon carbide omitted). GUS activity was quantified 
from approximately 0.1 microgram of TSP per sample in a 96-well white opaque microtiter plate using a three-point kinetic assay with $0.2 \mathrm{mM}$ 4-methylumbelliferyl $\beta$-Dglucuronide (MUG) as the substrate at $37^{\circ} \mathrm{C}$ [32]. Relative fluorescent measurements were converted into yield of GUS as a percent of total soluble protein based on assays of GUS type VII-A from E. coli (Sigma-Aldrich) as a standard.

\section{Leaf infiltrations}

Transient expression assays were performed using 4-5 week old $N$. benthamiana plants. Cultures of $A$. tumefaciens were prepared for leaf infiltrations as described for co-culture with plant tissue cultures with the following differences: cultures were washed and resuspended to a final $\mathrm{OD}_{600}$ of 1.2 in MMA media $\mathrm{pH}$ adjusted to 5.6 (10 mM MES (2-[N-morpholino] ethanesulfonic acid), $10 \mathrm{mM}$ magnesium chloride, $100 \mu \mathrm{M}$ acetosyringone). The cultures were then incubated for 2 hours at $25^{\circ} \mathrm{C}$ and pressure infiltrated into the abaxial surfaces of intact $N$. benthamiana leaves using a needleless $3 \mathrm{~mL}$ plastic syringe. Agroinfiltration was performed on the distal parts of leaves in the $5^{\text {th }}$ to $7^{\text {th }}$ tiers counting down from the first fully emerged leaf. At least four leaves on different plants were infiltrated for each treatment. Each leaf was infiltrated bilaterally with two independently harvested samples. For co-transformation of two or more A. tumefaciens clones containing different constructs, equal volumes of the individual cultures each at an $\mathrm{OD}_{600}$ of 1.2 were mixed together or diluted with MMA such that the final concentration of each clone was constant across comparative treatments. Control infiltrations comprised a mixture of pPSP19, pRep110 and MMA in a 1:1:1 ratio, or pPSP19 and MMA in a 1:1 ratio. Leaves were harvested after 6 days of co-cultivation except for the treatments containing pRep110, which were harvested after 5 days to avoid necrosis appearing at later times. Samples were harvested from each infiltration using a $1.9 \mathrm{~cm}$ diameter sharpened steel punch. The weight of each leaf disk was recorded (approximately $60 \mathrm{mg}$ ) and stored at $-20^{\circ} \mathrm{C}$ prior to quantitative GUS analysis.

\section{Vector construction}

The constructs used in this study are summarized in Figure 1. The GUS reporter consists of the uidA gene modified by the insertion of the potato PIV2 intron to prevent bacterial expression as described previously for the construction of pBY031-I1 and pGPTVK-GI [5]. Binary vectors pPSP19 and pRep110 contain the Tobacco bushy stunt virus $19 \mathrm{kDa}$ gene product (P19) and the native Bean yellow dwarf virus replication proteins, respectively [2]. Vector pHCPro contains the TEV P1/HC-Pro polyprotein as a HindIII fragment containing the dual $35 \mathrm{~S}$ expression cassette from pRTL2-0027 in the pGA482 binary vector [33].

The vector pGR106 [GenBank:AY297843] consists of a full-length PVX cDNA containing a duplicated coat protein subgenomic promoter and multiple cloning site (MCS) upstream of the coat protein gene [34]. The 5' end of the CP gene was removed from PGR106 by digestion with SalI and Xhol. Re-ligation of the compatible ends resulted in PVX $\triangle \mathrm{CP}$, which retains a 61 bp 3' terminal fragment of the $\mathrm{CP}$ important for viral replication [35]. Site-directed mutagenesis of PVX $\triangle C P$ was used to introduce a $3^{\text {rd }}$ Bsu36I recognition site at the 3 ' end of the triple gene block by removing the adenine residue at nucleotide 7647. A subsequent Bsu36I digestion and religation yielded PVX $\triangle \mathrm{CP} \triangle \mathrm{TGB}$ with a $1 \mathrm{~kb}$ deletion spanning the triple gene block. The GUS-intron coding sequence was PCR-amplified from pBY031-I1 using primers AscI_GI_F and GI_NotI_R (Table 1). The vector plasmids were sequentially digested with AscI and NotI while concurrently treated with shrimp alkaline phosphatase. The PCR-amplified insert was ligated into the AscI/NotI digested PVX vectors to create PVX-GUS, PVX $\triangle$ CP-GUS and PVX $\triangle \mathrm{CP} \Delta \mathrm{TGB}-\mathrm{GUS}$. Transformants were verified by colony PCR with primers Rep_F and GI_R. Amplified fragment sizes were $1.14 \mathrm{~kb}$ for PVX $\Delta$ CP $\Delta$ TGB-GUS and $2.13 \mathrm{~kb}$ for PVX $\Delta$ CP-GUS and PVX-GUS. The integrity of the plasmids was further verified by dual restriction digest with AscI and PstI.

Binary vectors containing the full-length cDNA of TRV isolate PpK20 RNA-1 (pTRV1) and RNA-2 (pYL156-GFP) were described previously [36,37]. The GUS-intron coding sequence with flanking NcoI and XhoI restriction was generated by PCR amplification of pBY031-I1 with primers Asc_GI_F and GI_XhoI_R. The corresponding NcoI-Xhol fragment containing the GFP ORF in pYL156-GFP was digested out and replaced with the GUS-intron fragment to create pTRV2-GUS. An undocumented NcoI recognition site present in the pYL156 vector backbone sequence necessitated a triple ligation due to the generation of two backbone fragments. Transformants were PCR-verified with primers PEBVsgPR_F and TRV3UTR_R. The integrity of the plasmid was further verified by restriction digest with SphI. The entire transcribed region of pTRV2-GUS including the 5' and 3' untranslated regions was verified by sequencing.

To create pEAQ-GUS the GUS-intron coding sequence was PCR amplified from pBY031-I1 with primers AgeI_GI_F and GI_XhoI_R. This fragment was then cloned into the AgeI and XhoI sites of the pEAQ-HT MCS such that the optional histidine tags were replaced. Transformants were PCR-verified with primers pEAQ_F and pEAQ_R to amplify a $2.56 \mathrm{~kb}$ fragment.

The high fidelity polymerases Phusion (New England Biolabs) or iProof (Bio-Rad) were used according to the 


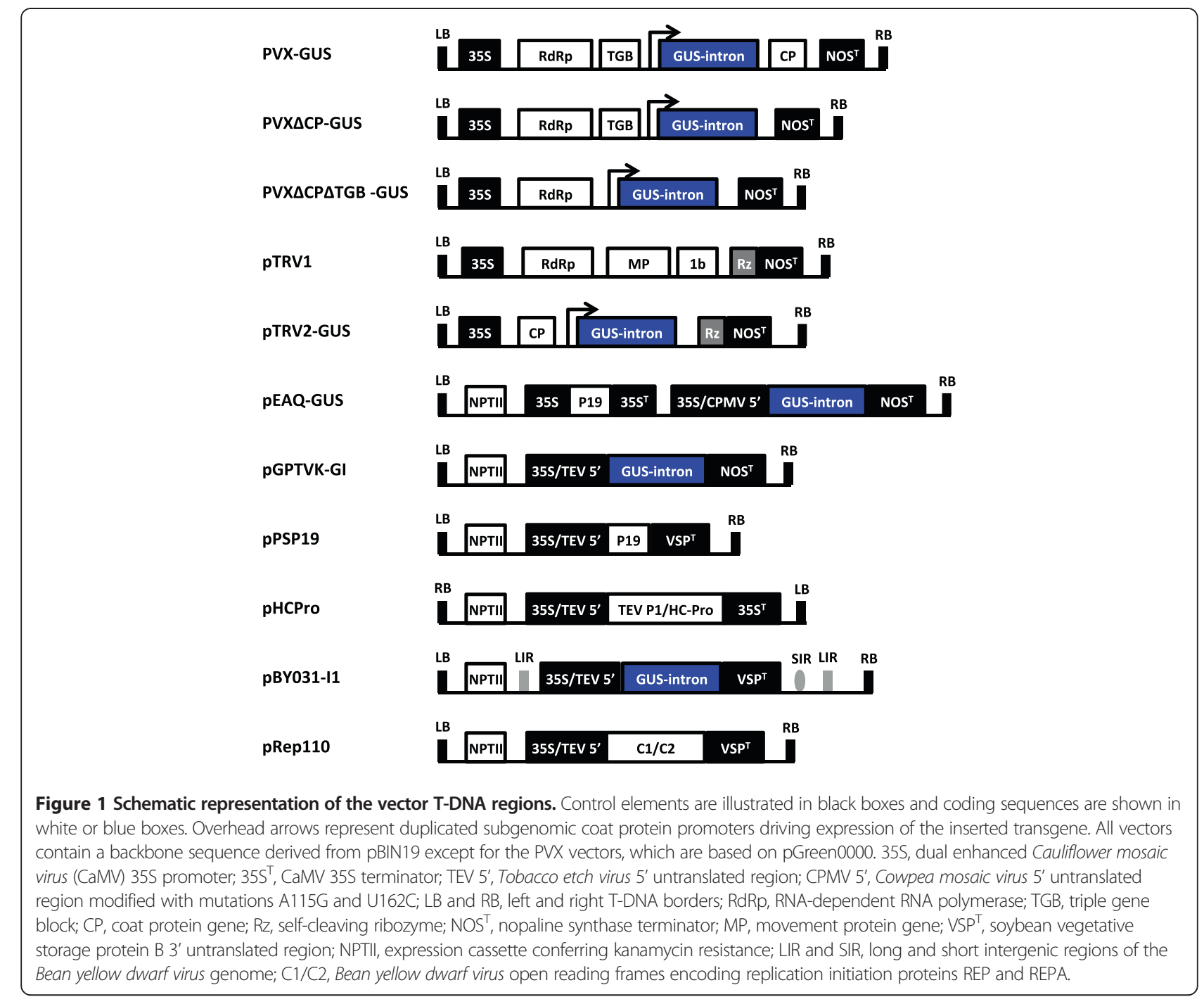

protocols suggested by the manufacturer to generate all cloning insert fragments. Ligation products were dropdialyzed on $13 \mathrm{~mm}$ diameter $0.025 \mu \mathrm{m}$ pore-size microdialysis membranes (MF-Millipore product code VSWP) prior to transformation into electrocompetent $E$. coli strains $\mathrm{DH} 5 \alpha$ or $\mathrm{DH} 10 \beta$ [38]. Electrocompetent E. coli and $A$. tumefaceins were prepared and transformed using the Bio-Rad MicroPulser Electroporation Apparatus as

Table 1 Sense (F) and antisense (R) oligonucleotides used for PCR amplifications

\begin{tabular}{|c|c|c|}
\hline Primer & Sequence $\left(5^{\prime}-3^{\prime}\right)$ & Hybridization site \\
\hline Ascl_Gl_F & TAGGCGCGCCATGGTCCGTCCTGTAGAA & $5^{\prime}$ terminus of GUS-intron \\
\hline Gl_Notl_R & TTGCGGCCGCAGAGGATCCTCATTGTTT & $3^{\prime}$ terminus of GUS-intron \\
\hline Rep_F & GCCTGAGTITTGTGGTTGG & internal to PVX RdRp \\
\hline$\overline{G l \_R}$ & GGATAGTCTGCCAGTTCAGTTCG & internal to GUS-intron \\
\hline Gl_Xhol_R & СCTACTCGAGCCTCATTGTTTGCCTCCC & 3' terminus of GUS-intron \\
\hline PEBVsgPR_F & AACTCGGTTTGCTGACCTAC & PEBV subgenomic promoter \\
\hline TRV3UTR_R & ACCTAAAACTTCAGACACGG & 3' UTR of TRV RNA-2 \\
\hline Agel-Gl_F & TATAACCGGTCATGGTCCGTCCTGTAGAAACC & $5^{\prime}$ terminus of GUS-intron \\
\hline pEAQ_F & AACGTTGTCAGATCGTGCTTCGGCACC & 5' UTR of pEAQ-HT \\
\hline$\overline{\mathrm{pEAQ} \_\mathrm{R}}$ & CTCCTGTTTAGCAGGTCGTCCCTTCAG & $3^{\prime}$ UTR of pEAQ-HT \\
\hline
\end{tabular}

Sequences added with $5^{\prime}$ extensions are shown in bold with restriction enzyme recognition sites underlined. Initiation codons are italicized. UTR, untranslated region; PEBV, Pea early browning virus; RdRp, RNA-dependent RNA polymerase. 
per the manufacturer's protocols. Plasmid pGR106 and its derivatives were co-transformed with the pSoup helper plasmid [39]. All A. tumefaciens and E. coli transformants were selected on LB medium supplemented with $50 \mathrm{mg} / \mathrm{L}$ kanamycin and incubated at $25^{\circ} \mathrm{C}$ and $37^{\circ} \mathrm{C}$, respectively. All A. tumefaciens transformants were colony purified and subsequently verified by colony PCR.

\section{Statistical analysis}

All probabilities were calculated using Welch's $t$ test with a two-tailed distribution assuming unequal variance between data sets consisting of at least three replicates.

\section{Results}

In cell suspensions, a replicating PVX vector yielded 6.6-fold more GUS when non-essential viral genes were omitted

In the present study three different PVX constructs were characterized: a PVX full-length vector containing GUS as a gene insert (PVX-GUS) and derivatives of that vector containing deletions spanning most of the coat protein (PVX $\triangle \mathrm{CP}-\mathrm{GUS}$ ) or both the coat protein and triple gene block (PVX $\triangle \mathrm{CP} \triangle \mathrm{TGB}-\mathrm{GUS})$. The latter vector is referred to as a minimal vector since it retains only the viral components required for replication, including the RNAdependent RNA polymerase. In all cases a plant intron within the GUS gene prevents bacterial expression. GUS production by $A$. tumefaciens has been demonstrated from a deleted vector of Foxtail mosaic virus, a potexvirus closely related to PVX [40].

In cell suspensions the PVX movement functions encoded by the TGB and CP genes were dispensable when complemented by the efficient viral cDNA delivery capabilities of $A$. tumefaciens. Transient GUS expression was 6.6-fold higher with the minimal vector relative to the full-length vector (Figure 2A). Expression from the $\mathrm{CP}$-replacement vector was intermediate between the full-length and minimal vectors and significantly different from both. Thus, vectors lacking non-essential viral genes were free to direct more of the cellular metabolic resources towards the production of the target protein. Relative to cell suspensions, average transient GUS expression from the minimal PVX vector was 244-fold lower in hairy roots, where the presence of the CP and TGB genes had an insignificant effect (Figure 2B).

The relative performance of the PVX vectors in cell suspensions is consistent with previous reports of similar vectors in leaves. Omission of the PVX CP gene led to higher target protein expression in infiltrated $N$. benthamiana leaves [41]. Similarly, a minimal PVX vector (PVXdt-GFP) lacking both the CP and TGB genes yielded 2.5-fold more transiently expressed GFP relative to a full-length vector when co-expressed with HC-Pro [35].

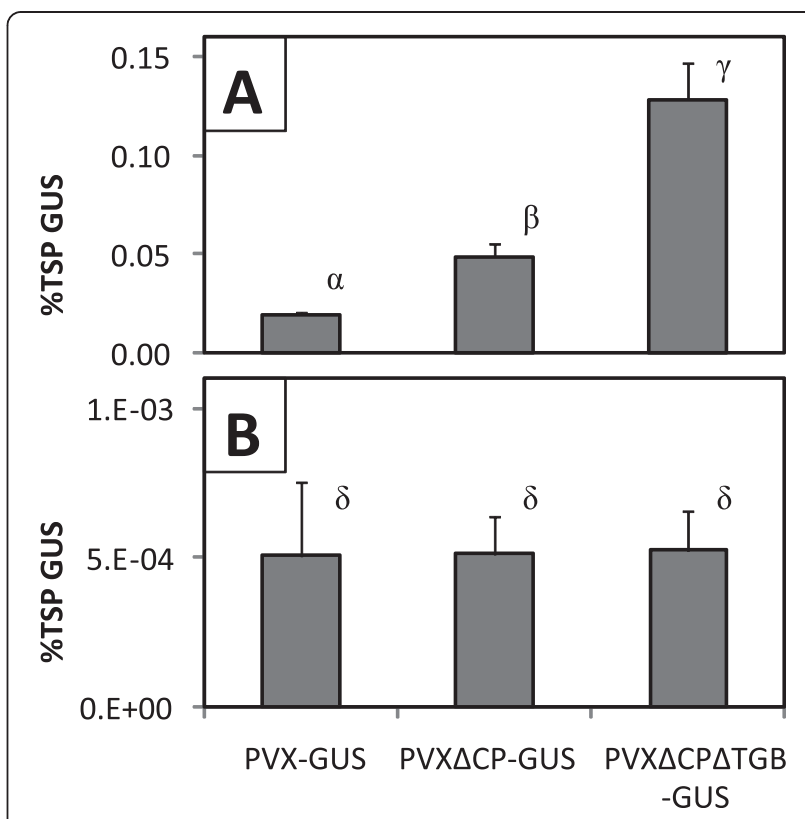

Figure 2 Transient GUS expression from PVX vectors in cell suspensions and hairy roots. The PVX-GUS vector containing GUS as an insert driven by a duplicated coat protein subgenomic promoter was compared to a coat protein replacement construct (PVX $\triangle C P-G U S$ ) and a minimal vector lacking the coat protein and the triple gene block (PVX $\triangle C P \triangle T G B-G U S$ ) in (A) cell suspensions, and (B) hairy roots. Values represent the mean \pm SD of samples from three co-cultures. Means labeled with the same Greek letter are not different from each other at the $95 \%$ significance level.

\section{Suppression of post-transcriptional gene silencing} increased transient GUS expression from the minimal PVX vector in cell suspensions

The minimal PVX vector contains a deletion in the triple gene block that includes the P25 gene encoding the native suppressor of PTGS [42]. Thus, we sought to potentially complement this disrupted function through co-expression of P19. Co-transformation of the minimal vector (PVX $\triangle \mathrm{CP} \triangle \mathrm{TGB}-\mathrm{GUS}$ ) with pPSP19 increased transient GUS expression by $44 \%$ and $990 \%$ in co-cultured cell suspensions (Figure 3A) and infiltrated leaves (Figure 3B), respectively. This increase was statistically significant in leaves but not cell suspensions. A similar trend was observed for transient co-expression of the HC-Pro suppressor of Tobacco etch virus, which led to a statistically significant $83 \%$ increase in transient GUS expression in cell suspensions $(\mathrm{p}<0.05$; Figure $3 \mathrm{~A})$. Co-transformation with pHCPro also increased transient GUS expression in leaves, but severe necrosis present at six days post-infiltration precluded accurate quantification. Similar results were observed with a non-replicating expression vector: Cotransformation of pGPTVK-GI with pPSP19 increased transient GUS expression by $184 \%$ in cell suspensions (Figure 4A). 


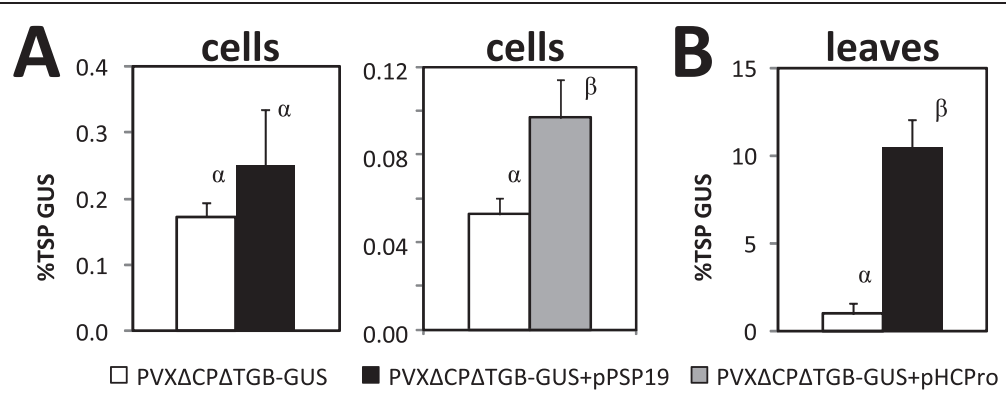

Figure 3 Suppression of post-transcriptional gene silencing in cell suspensions and leaves. The minimal PXV vector (PVX $\triangle C P \triangle T G B-G U S)$ was transiently transformed alone or co-transformed with a suppressor of PTGS (either pPSP19 or pHCPro). Values represent the mean \pm SD of samples from (A) three cell suspension co-cultures, and (B) three infiltrated leaves of intact plants. Means on the same axis labeled with the same Greek letter are not different from each other at the $95 \%$ significance level.

Consistent with the results of the present study, $N$. benthamiana leaves infiltrated with PVXdt-GFP accumulated more GFP when HC-Pro was co-expressed [35]. Similarly, co-transformation of a non-replicating conventional vector with a P19 construct significantly increased peak transient expression of an IgG antibody by $75 \%$ and $1300 \%$ in co-cultured $N$. benthamiana cell suspensions and infiltrated leaves, respectively [43].

\section{A non-replicating vector containing a leader sequence from Cowpea mosaic virus modified for enhanced translation led to higher transient GUS expression in cell suspensions}

Relative to the control treatment, pEAQ-GUS resulted in approximately $70 \%$ higher transient GUS expression in both co-cultured cell suspensions and infiltrated leaves (Figure 4A). The increase in expression was statistically significant in leaves but not cell suspensions. Average transient GUS expression from the pEAQ-GUS vector in cell suspensions $(0.51 \%$ TSP) was consistent with the only other report pertaining to the use of the CPMV-HT expression system in this host. Tobacco Bright Yellow-2 cells stably transformed with pEAQ-SP-HSA expressed recombinant human serum albumin (rHSA) at $11.88 \mathrm{mg} / \mathrm{L}$ in the culture medium, accounting for $0.7 \%$ TSP. In contrast a conventional CaMV 35S-based vector (lacking P19) failed to yield detectable quantities of secreted rHSA [44].

\section{A TRV vector capable of systemic movement outperformed the analogous PVX vector in hairy roots} The pTRV2-GUS vector yielded 150-fold more GUS than PVX-GUS (Table 2). Thus the results of the present study extend the root tropism of TRV to in vitro cultures of hairy roots. In fact, all other vectors tested to date have proven to be unsuitable for high-level transient expression: Hairy roots infected with the TRV vector expressed at least an order of magnitude more GUS than the highest level previously reported with the replicating pBY031-I1 vector derived from Bean yellow dwarf virus. Thus TRV yielded transient expression levels in hairy
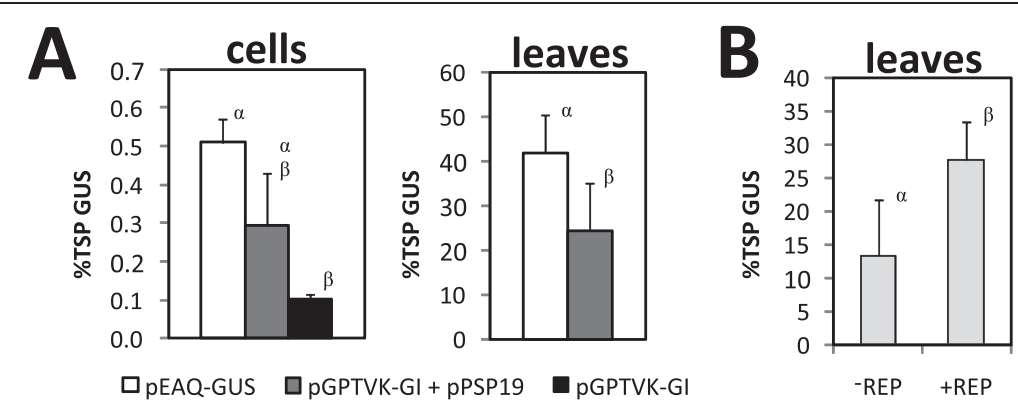

Figure 4 Transient GUS expression from non-replicating and replicating viral vectors in cell suspensions and leaves. (A) Non-replicating vectors contained the GUS-intron gene downstream of a conventional TEV 5'UTR (pGPTVK-GI) or a modified CPMV 5' UTR (pEAQ-GUS). The latter vector provided P19 as a second expression cassette on a single T-DNA. Values represent the mean \pm SD of samples from three co-cultures or four infiltrated leaves. (B) The replication-competent vector PBY031-I1 and PPSP19 were co-delivered to N. benthamiana leaves together (-REP) or with a third binary vector containing the Bean yellow dwarf virus DNA replicase gene (+REP). Values represent the mean \pm SD of samples from seven leaf infiltrations on different leaves. Means on the same axis labeled with the same Greek letter are not different from each other at the $95 \%$ significance level. 
Table 2 Comparison of different viral vectors used for transient GUS expression in hairy roots

\begin{tabular}{lllll}
\hline Construct & Species & $\begin{array}{l}\text { GUS } \\
\text { (\%TSP) }\end{array}$ & Std. dev. & $\begin{array}{l}\text { Days in } \\
\text { co-culture }\end{array}$ \\
\hline pTRV2-GUS & N. benthamiana & 0.076 & 0.027 & 6 \\
\hline PVX-GUS & N. benthamiana & 0.0005 & 0.0002 & 6 \\
\hline pEAQ-GUS & N. benthamiana & 0.006 & 0.004 & 4 \\
\hline pBY031-I1 & N. glutinosa & 0.007 & 0.003 & 3 \\
\hline
\end{tabular}

roots that are on par with those previously observed only in plant cell suspensions, which typically exceed levels in hairy roots by 20 -fold for vectors lacking viral movement functions [5]. Even more remarkable is that this magnitude of expression reflected that obtained from a non-optimized process. Simply initiating the co-culture 7 rather than 5 days after hairy root culture initiation significantly increased average transient GUS expression by more than 4 -fold to $0.5 \%$ TSP (Figure $5 \mathrm{~A}$ ).

\section{Transient GUS expression in hairy roots underestimates} the capacity for heterologous protein production

For a movement-enabled vector such as TRV, transient GUS expression is expected in both cells directly colonized and transiently transformed by $A$. tumefaciens as well as distal cells susceptible to secondary infection. Primary infection occurs in actively dividing surface cells that are accessible by $A$. tumefaciens and amenable to T-DNA transfer such as adventitious laterals, root tips and the root zone of elongation [5]. Cell-to-cell and systemic viral movement lead to secondary infections that occur over a period of days following primary infection.

Histochemical staining of hairy roots co-cultured with pTRV2-GUS revealed a heterogeneous pattern of GUS expression (Figure 5B). Heterogeneous expression implies that there is room for improving productivity per unit biomass because bulk samples are diluted by nontransformed tissue. Thus the reported expression levels do not reflect the capacity for expression on the cellular level. Simply extending the duration of the co-culture did not increase qualitative GUS expression, suggesting that the kinetics of viral movement were not limiting (results not shown). Instead, it appears that this combination of viral vector and host tissue may be suboptimal.

\section{Discussion}

There have been several reports of RNA viruses engineered for high-level heterologous protein expression in cell suspensions and hairy roots. The highest reported level of heterologous protein expression from a viral vector in plant tissue culture was achieved with an estrogeninducible promoter fused to the cDNA of a Tomato mosaic virus $\mathrm{CP}$-replacement vector. A tobacco BY-2 cell suspension stably transformed with this vector accumulated GFP to 10\% TSP [45]. A full-length Tobacco mosaic virus (TMV) vector has also been used to transiently express GFP in $N$. benthamiana cell suspensions and hairy roots co-incubated with infectious recombinant viral particles. The recombinant virus failed to replicate or lead

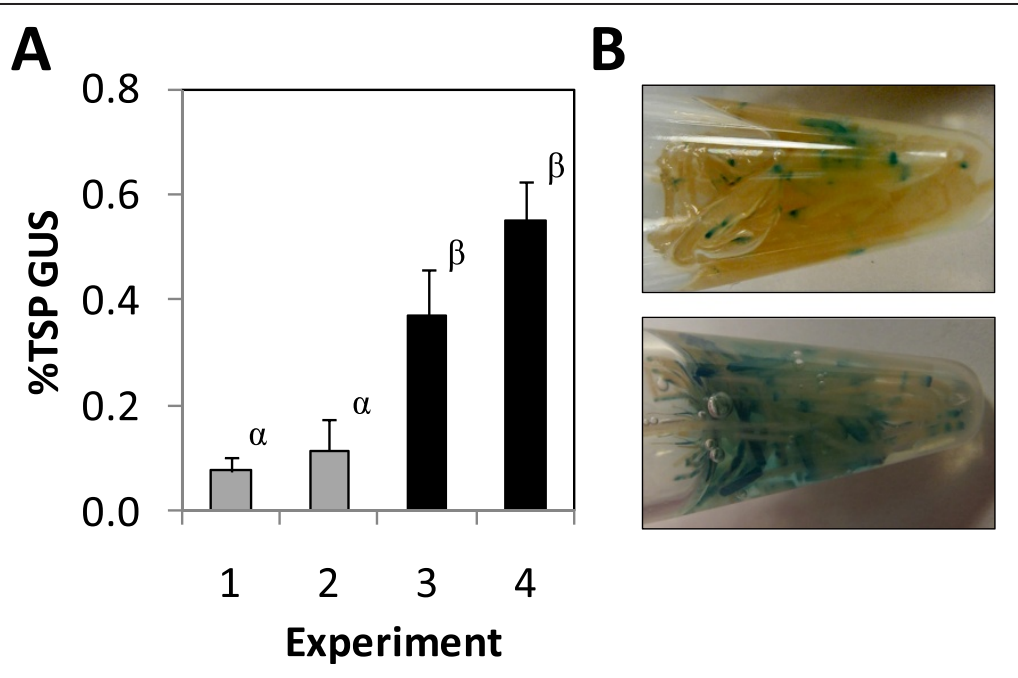

Figure 5 Transient GUS expression from a TRV vector in hairy roots. (A) Hairy root cultures were initiated and incubated for 5 (gray bars) or 7 days (black bars) prior to co-culture with A. tumefaciens clones harboring pTRV1 and pTRV2-GUS. Values represent the mean \pm SD of three cocultures harvested six days after initiation. Four separate experiments were conducted over a period of 13 months. Means labeled with the same Greek letter are not different from each other at the 95\% significance level. (B) Histochemical detection of transient GUS expression in representative samples collected from Experiments 1 (top) and 4 (bottom). Hairy roots were incubated with X-Gluc substrate in $1.5 \mathrm{~mL}$ microcentrifuge tubes for 60 minutes at room temperature. Areas stained blue are positive for GUS expression. 
to significant GFP accumulation in hairy roots due to foreign gene deletion that occurred during serial passaging of the recombinant TMV stocks in plants [46]. Similarly, very limited uptake and replication was observed for infectious recombinant TMV particles co-incubated with cell suspensions [47]. In an alternative approach, hairy roots of $N$. benthamiana were established from the leaves of plants previously infected with the same recombinant TMV vector. These clonal hairy root lines sustained recombinant virus replication and GFP expression over a period of three years in the absence of selection at levels comparable to those found in transgenic hairy roots [48].

In contrast, our approach of harnessing an engineered strain of $A$. tumefaciens to amplify and deliver viral cDNA to pre-established plant tissue cultures avoids the instability and long lead times associated with viral passaging in whole plants or establishing recombinant virus-infected root lines from infected leaves. Moreover, viral cDNA transfection by $A$. tumefaciens in plant tissue cultures may be more efficient and reproducible than passive uptake of viral particles. Agroinfiltration led to almost complete infection in leaves even with TMV viral vectors disabled for cell-to-cell movement [49].

The relatively low expression we consistently observed in cell suspensions (approximately $0.5 \%$ TSP, or $3 \mathrm{mg} / \mathrm{L}$ ) was not the result of incompatibilities of the viral vectors with this host (N. benthamiana). Viral vectors of disparate origin that rely on three distinct mechanisms of transgene amplification (DNA replication for Bean yellow dwarf virus, mRNA replication for PVX, and hyper-translation for CPMV-HT) demonstrated high-level expression in leaves, but not cell suspensions. For example, using a DNA viral vector derived from Bean yellow dwarf virus, transient GUS expression was 125-folder higher in $N$. benthamiana leaves (27.6\% TSP; Figure 4B) relative to $N$. glutinosa cells $(0.22 \%$ TSP; [5]). In both hosts complementation of pBY031-I1 with the viral replicase proteinsupplying vector (pRep110) had the effect of doubling relative GUS expression levels, demonstrating functional viral amplification abilities. Moreover, pGPTVK-GI, pEAQGUS and PVX $\triangle \mathrm{CP} \triangle \mathrm{TGB}-\mathrm{GUS}$ yielded 70 to 80 -fold more GUS in leaves relative to cells in the presence of P19. Likewise, transient co-expression of a murine IgG1 with P19 was reported to be 75 -fold higher in infiltrated $N$. benthamiana leaves relative to cell suspensions [43]. These results are inconsistent with the commonly held notion that the application of viral vectors and/or suppression of gene silencing may readily overcome the low titers characteristic of transient expression in cell suspensions.

The unique physiology of cell suspensions may account for the observed lower absolute expression levels that were recalcitrant to suppression of post-transcriptional gene silencing. Relative to cell suspensions, mature leaves may provide a more natural environment for the attachment of A. tumefaciens and subsequent transfer of T-DNA. Yet, the highest yields for transient expression have been achieved in heterotrophic plant cell suspensions undergoing rapid cell division, whereas non-dividing but metabolically active cells in fully expanded leaves tend to work best in planta [50,51]. Consequently, differences in physiological state other than cell cycle status must play an important role in mediating transient protein expression of ectopic DNA.

Plant cell suspensions are composed of individual or small aggregates of dedifferentiated cells that lack fully functional plasmodesmata due to minimal cell-cell contacts. Hence, systemic PTGS may be reduced since the silencing signal is generally transmitted via plasmodesmata and the vascular system [52,53]. Protein degradation in cell suspensions has been recognized to play an important role in limiting overall heterologous protein yields to less than 1\% TSP [54]. The intracellular concentration of a protein reflects a balance between its rate of synthesis and degradation. Thus, strategies to amplify protein synthesis through upstream processes including transcription and translation may be negated or obscured by the effect of increased protein turnover. Moreover, this protein degradation is likely non-specific since GUS is highly stable within the cytoplasmic environment and has even been used as a protein-stabilizing fusion partner $[55,56]$. Co-expression of a heterologous protease inhibitor represents a potential method to down-regulate protein turnover and increase the accumulation of target protein in cell suspensions [57].

\section{Conclusions}

To the best of the authors' knowledge this is the first report of the application of RNA viral vectors for Agrobacterium-mediated transient protein expression in plant tissue culture. Furthermore, the TRV vector described here represents the first and only vector to achieve transient expression levels in hairy roots comparable to those observed in plant cell suspensions using this method. Despite equivalent or superior performance in side-by-side comparisons with non-viral vectors in plant cell suspensions, the PVX and CPMV-HT vectors did not overcome the batch-to-batch variability that characterizes transient protein expression in this platform. However, the reported trends were reproducible across independent experiments notwithstanding these fluctuations in absolute expression levels.

Our future work will focus on engineering hairy root lines with integrated suppression of PTGS and developing TRV vectors with increased infectivity. Hairy roots transformed to constitutively express a strong suppressor of PTGS may support higher levels of transient protein expression because the native $16 \mathrm{kDa}$ gene product of TRV is relatively weak compared to P19 of Tomato 
bushy stunt virus [58]. Optimization of a TMV vector by removal of putative splice sites and insertion of multiple plant introns increased the efficiency of viral replication initiation by up to three orders of magnitude in $N$. benthamiana infiltrated leaves [49]. Additionally, a modified TRV expression vector retaining the native $2 \mathrm{~b}$ gene on RNA-2, required for nematode transmission, displayed increased infectivity and root meristem invasion in $N$. benthamiana plants [24]. Similar modifications to pTRV1 and pTRV2-GUS could improve the efficiency of primary (nuclear-launched) infection and systemic movement. We anticipate that the implementation of these improvements to this vector and host combination will yield the high and homogeneous expression characteristic of infiltrated leaves.

\section{Abbreviations \\ PVX: Potato virus X; TRV: Tobacco rattle virus; GUS: $\beta$-glucuronidase; CPMV: Cowpea mosaic virus; PTGS: Post-transcriptional gene silencing; ORF: Open reading frame; TGB: Triple gene block; CP: Coat protein; TSP: Total soluble protein; TEV: Tobacco etch virus; MCS: Multiple cloning site; GFP: Green fluorescent protein; TMV: Tobacco mosaic virus.}

\section{Competing interests}

The authors declare that they have no competing interests.

\section{Acknowledgements}

The authors acknowledge Hugh Mason for providing PPSP19 and David Baulcombe for the PVX construct pGR106. Vicki Vance and Gail Pruss are acknowledged for their generous contributions of the PVX $\triangle C P$ and PVX $\triangle$ CPATGB derivatives of pGR106 as well as the pHCPro construct. Michael Axtell kindly provided the pSoup helper plasmid. The authors thank Mark Varrelmann for providing PYL156-GFP and George Lomonossoff and Frank Sainsbury for providing pEAQ-HT. pTRV1 was obtained from The Arabidopsis Biological Resource Center. Nicotiana spp. seeds were obtained from the USDA Germplasm Resources Information Network. This work was supported in part by the National Science Foundation under grant no. BCS-0003926 and GOALI Program. Any opinions, findings, and conclusions or

recommendations expressed in this material are those of the authors and do not necessarily reflect the views of the National Science Foundation.

\section{Authors' contributions}

JSL was responsible for experiment design, execution, analysis and interpretation, and wrote the manuscript. WRC conceived the study, oversaw the experimental work, contributed to interpretation, and edited the manuscript. Both authors read and approved the final manuscript.

Received: 16 January 2012 Accepted: 6 May 2012

Published: 6 May 2012

\section{References}

1. Sainsbury F, Lomonossoff GP: Extremely high-level and rapid transient protein production in plants without the use of viral replication. Plant Physiol 2008, 148(3):1212-1218.

2. Huang Z, Chen Q, Hjelm B, Arntzen C, Mason H: A DNA replicon system for rapid high-level production of virus-like particles in plants. Biotechnol Bioeng 2009, 103(4):706-714.

3. Musiychuk K, Stephenson N, Bi H, Farrance CE, Orozovic G, Brodelius M, Brodelius P, Horsey A, Ugulava N, Shamloul AM, Mett V, Rabindran S, Streatfield SJ, Yusibov V: A launch vector for the production of vaccine antigens in plants. Influenza Other Respi Viruses 2007, 1(1):19-25.

4. Lindbo JA: TRBO: a high-efficiency tobacco mosaic virus RNA-based overexpression vector. Plant Physiol 2007, 145(4):1232-1240.

5. Collens JI, Mason HS, Curtis WR: Agrobacterium-mediated viral vectoramplified transient gene expression in Nicotiana glutinosa plant tissue culture. Biotechnol Prog 2007, 23(3):570-576.
6. Fischer R, Schillberg S, Hellwig S: Twyman RM. Drossard J: GMP issues for recombinant plant-derived pharmaceutical proteins. Biotechnol Adv 2011, 30(2):434-439.

7. Weathers PJ, Towler MJ, Xu J: Bench to batch: advances in plant cell culture for producing useful products. Appl Microbiol Biotechnol 2010, 85 (5):1339-1351.

8. Ritterhaus E, Ulrich J, Westphal K: Large-scale production of plant cell cultures. Int Assoc Plant Tissue Cult Newsl 1990, 61:2-10.

9. Hsiao TY, Bacani FT, Carvalho EB, Curtis WR: Development of a low capital investment reactor system: application for plant cell suspension culture. Biotechnol Prog 1999, 15(1):114-122.

10. Shanks JV, Morgan J: Plant 'hairy root' culture. Curr Opin Biotechnol 1999, 10(2):151-155

11. Ramakrishnan D, Curtis WR: Trickle-bed root culture bioreactor design and scale-up: growth, fluid-dynamics, and oxygen mass transfer. Biotechnol Bioeng 2004, 88(2):248-260.

12. D'Aoust MA, Couture MM, Charland N, Trepanier S, Landry N, Ors F, Vezina LP: The production of hemagglutinin-based virus-like particles in plants: a rapid, efficient and safe response to pandemic influenza. Plant Biotechnol J 2010, 8(5):607-619.

13. Kiselev KV, Turlenko AV, Tchernoded GK, Zhuravlev YN: Nucleotide substitutions in rolC and nptll gene sequences during long-term cultivation of Panax ginseng cell cultures. Plant Cell Rep 2009, 28(8):1273-1278.

14. Roth BM, Pruss GJ, Vance VB: Plant viral suppressors of RNA silencing. Virus Res 2004, 102(1):97-108.

15. Voinnet $\mathrm{O}$, Rivas $\mathrm{S}$, Mestre $\mathrm{P}$, Baulcombe D: An enhanced transient expression system in plants based on suppression of gene silencing by the p19 protein of tomato bushy stunt virus. Plant J 2003, 33(5):949-956.

16. Batten JS, Yoshinari S, Hemenway C: Potato virus $X$ : a model system for virus replication, movement and gene expression. Molecular Plant Pathology 2003, 4(2):125-131.

17. Chapman S, Kavanagh T, Baulcombe D: Potato virus $\mathrm{X}$ as a vector for gene expression in plants. The Plant J 1992, 2(4):549-557.

18. Azhakanandam K, Weissinger SM, Nicholson JS, Qu R, Weissinger AK: Amplicon-plus targeting technology (APTT) for rapid production of a highly unstable vaccine protein in tobacco plants. Plant Mol Biol 2007, 63 (3):393-404

19. Cerovska N, Hoffmeisterova H, Pecenkova T, Moravec T, Synkova H, Plchova $H$, Veleminsky J: Transient expression of HPV16 E7 peptide (aa 44-60) and HPV16 L2 peptide (aa 108-120) on chimeric potyvirus-like particles using Potato virus X-based vector. Protein Expr Purif 2008, 58(1):154-161.

20. Mechtcheriakova IA, Eldarov MA, Nicholson L, Shanks M, Skryabin KG Lomonossoff GP: The use of viral vectors to produce hepatitis B virus core particles in plants. J Virol Methods 2006, 131(1):10-15.

21. Manske U, Schiemann J: Development and assessment of a potato virus $\mathrm{X}$-based expression system with improved biosafety. Environ Biosafety Res 2005, 4(1):45-57.

22. MacFarlane SA: Molecular biology of the tobraviruses. $J$ Gen Virol 1999, 80:2799-2807.

23. MacFarlane SA, Popovich AH: Efficient expression of foreign proteins in roots from tobravirus vectors. Virology 2000, 267(1):29-35.

24. Valentine T, Shaw J, Blok VC, Phillips MS, Oparka KJ, Lacomme C: Efficient virus-induced gene silencing in roots using a modified tobacco rattle virus vector. Plant Physiol 2004, 136(4):3999-4009.

25. Sainsbury F, Thuenemann EC, Lomonossoff GP: pEAQ: versatile expression vectors for easy and quick transient expression of heterologous proteins in plants. Plant Biotechnol J 2009, 7(7):682-693.

26. Sainsbury F, Sack M, Stadlmann J, Quendler H, Fischer R, Lomonossoff GP: Rapid transient production in plants by replicating and non-replicating vectors yields high quality functional anti-HIV antibody. PLoS One 2010, 5(11):e13976.

27. Murashige T, Skoog F: A revised medium for rapid growth and bioassays with tobacco tissue cultures. Physiol Plant 1962, 15:473-497.

28. Gamborg OL, Miller RA, Ojima K: Nutrient requirements of suspension cultures of soybean root cells. Exp Cell Res 1968, 50(1):151-158.

29. Collens JI, Lee DR, Seeman AM, Curtis WR: Development of auxotrophic Agrobacterium tumefaciens for gene transfer in plant tissue culture. Biotechnol Prog 2004, 20(3):890-896.

30. Stomp A: Histochemical localization of [beta]-glucuronidase. In In GUS Protocols. Edited by Gallagher SR. San Diego, CA: Academic; 1992:103-113.

31. Zor T, Selinger Z: Linearization of the Bradford protein assay increases its sensitivity: theoretical and experimental studies. Anal Biochem 1996, 236(2):302-308. 
32. Rao AG, Flynn P: A microtiter plate-based assay for beta-D-glucuronidase: A quantitative approach. In In GUS Protocols. Edited by Gallagher SR. San Diego, CA: Academic; 1992:89-99.

33. Carrington JC, Freed DD, Oh CS: Expression of potyviral polyproteins in transgenic plants reveals three proteolytic activities required for complete processing. EMBO J 1990, 9(5):1347-1353.

34. Lu R, Malcuit I, Moffett P, Ruiz MT, Peart J, Wu AJ, Rathjen JP, Bendahmane A, Day L, Baulcombe DC: High throughput virus-induced gene silencing implicates heat shock protein 90 in plant disease resistance. EMBO J 2003, 22(21):5690-5699.

35. Komarova TV, Skulachev MV, Zvereva AS, Schwartz AM, Dorokhov YL, Atabekov JG: New viral vector for efficient production of target proteins in plants. Biochemistry (Mosc) 2006, 71(8):846-850.

36. Liu Y, Schiff M, Marathe R, Dinesh-Kumar SP: Tobacco Rar1, EDS1 and NPR1/NIM1 like genes are required for N-mediated resistance to tobacco mosaic virus. Plant J 2002, 30(4):415-429.

37. Ghazala W, Varrelmann M: Tobacco rattle virus $29 \mathrm{~K}$ movement protein is the elicitor of extreme and hypersensitive-like resistance in two cultivar of Solanum tuberosum. Mol Plant Microbe Interact 2007, 20(11):1396-1405

38. Drop Dialysis [http://www.neb.com/nebecomm/techBulletinFiles/ DROP_Dialysis.pdf]

39. Hellens RP, Edwards EA, Leyland NR, Bean S, Mullineaux PM: pGreen: a versatile and flexible binary $\mathrm{Ti}$ vector for Agrobacterium-mediated plant transformation. Plant Mol Biol 2000, 42(6):819-832.

40. Liu Z, Kearney CM: An efficient Foxtail mosaic virus vector system with reduced environmental risk. BMC Biotechnol 2010, 10(1):88

41. Giritch A, Marillonnet S, Engler C, van Eldik G, Botterman J, Klimyuk V, Gleba Y: Rapid high-yield expression of full-size lgG antibodies in plants coinfected with noncompeting viral vectors. Proc Natl Acad Sci U S A 2006 103(40):14701-14706.

42. Voinnet $\mathrm{O}$, Lederer $\mathrm{C}$, Baulcombe DC: A viral movement protein prevents spread of the gene silencing signal in Nicotiana benthamiana. Cell 2000, 103(1):157-167.

43. Boivin EB, Lepage E, Matton DP, De Crescenzo G, Jolicoeur M: Transient expression of antibodies in suspension plant cell suspension cultures is enhanced when co-transformed with the tomato bushy stunt virus $\mathrm{p} 19$ viral suppressor of gene silencing. Biotechnol Prog 2010, 26(6):1534-1543.

44. Sun QY, Ding LW, Lomonossoff GP, Sun YB, Luo M, Li CQ, Jiang L, Xu ZF: Improved expression and purification of recombinant human serum albumin from transgenic tobacco suspension culture. J Biotechnol 2011, 155(2):164-172.

45. Dohi K, Nishikiori M, Tamai A, Ishikawa M, Meshi T, Mori M: Inducible virusmediated expression of a foreign protein in suspension-cultured plant cells. Arch Virol 2006, 151(6):1075-1084.

46. Shadwick FS, Doran PM: Propagation of plant viruses in hairy root cultures: a potential method for in vitro production of epitope vaccines and foreign proteins. Biotechnol Bioeng 2007, 96(3):570-583.

47. Shih SM, Doran PM: In vitro propagation of plant virus using different forms of plant tissue culture and modes of culture operation. J Biotechnol 2009, 143(3):198-206.

48. Skarjinskaia M, Karl J, Araujo A, Ruby K, Rabindran S, Streatfield SJ, Yusibov V: Production of recombinant proteins in clonal root cultures using episomal expression vectors. Biotechnol Bioeng 2008, 100(4):814-819.

49. Marillonnet S, Thoeringer C, Kandzia R, Klimyuk V, Gleba Y: Systemic Agrobacterium tumefaciens-mediated transfection of viral replicons for efficient transient expression in plants. Nat Biotechnol 2005, 23(6):718-723.

50. O'Neill KM, Larsen JS, Curtis WR: Scale-up of Agrobacterium-mediated transient protein expression in bioreactor-grown Nicotiana glutinosa plant cell suspension culture. Biotechnol Prog 2008, 24(2):372-376.

51. Sheludko YV, Sindarovska YR, Gerasymenko IM, Bannikova MA, Kuchuk NV: Comparison of several Nicotiana species as hosts for high-scale Agrobacterium-mediated transient expression. Biotechnol Bioeng 2007, 96(3):608-614

52. Crawford KM, Zambryski PC: Plasmodesmata signaling: many roles, sophisticated statutes. Curr Opin Plant Biol 1999, 2(5):382-387.

53. Doran PM: Foreign protein production in plant tissue cultures. Curr Opin Biotechnol 2000, 11(2):199-204.

54. Doran PM: Foreign protein degradation and instability in plants and plant tissue cultures. Trends Biotechnol 2006, 24(9):426-432.
55. Gil F, Brun A, Wigdorovitz A, Catala R, Martinez-Torrecuadrada JL, Casal I, Salinas J, Borca MV, Escribano JM: High-yield expression of a viral peptide vaccine in transgenic plants. FEBS Lett 2001, 488(1-2):13-17.

56. Escribano JM, Perez-Filgueira DM: Strategies for improving vaccine antigens expression in transgenic plants: fusion to carrier sequences. Methods Mol Biol 2009, 483:275-287.

57. Goulet C, Benchabane M, Anguenot R, Brunelle F, Khalf M, Michaud D: A companion protease inhibitor for the protection of cytosol-targeted recombinant proteins in plants. Plant Biotechnol J 2010, 8(2):142-154.

58. Martin-Hernandez AM, Baulcombe DC: Tobacco rattle virus 16-kilodalton protein encodes a suppressor of RNA silencing that allows transient viral entry in meristems. J Virol 2008, 82(8):4064-4071.

doi:10.1186/1472-6750-12-21

Cite this article as: Larsen and Curtis: RNA viral vectors for improved Agrobacterium-mediated transient expression of heterologous proteins in Nicotiana benthamiana cell suspensions and hairy roots. BMC Biotechnology 2012 12:21.

\section{Submit your next manuscript to BioMed Central and take full advantage of:}

- Convenient online submission

- Thorough peer review

- No space constraints or color figure charges

- Immediate publication on acceptance

- Inclusion in PubMed, CAS, Scopus and Google Scholar

- Research which is freely available for redistribution 